\title{
Effects of a Mobile-App-Based Self-Management Support Program For Elderly Hemodialysis Patients
}

\author{
Youngsoon Min ${ }^{1}$, Myonghwa Park ${ }^{2}$ \\ ${ }^{1}$ Hemodialysis Unit, Chungnam National University Hospital, Daejeon, Korea \\ ${ }^{2}$ College of Nursing, Chungnam National University, Daejeon, Korea
}

Objectives: To identify the effects of a mobile-app-based self-management program for elderly hemodialysis patients on their sick-role behavior, basic psychological needs, and self-efficacy. Methods: A nonequivalent control group with a nonsynchronized design was utilized, and 60 participants (30 in each of the experimental and control groups) were recruited from Chungnam National University Hospital from March to August 2018. The program consisted of continuous training on how to use the mobile-app, self-checking via the app, message transfer through Electronic Medical Records, and feedback. The control group received the usual care. Data were analyzed using the $\chi^{2}$-test, the $t$-test, the repeated-measures ANOVA, and the McNemar test. A formalized messaging program was developed, and the app was developed with consideration of the specific physical and cognitive limitations of the elderly. Results: Comparisons were conducted between the experimental $(n=28)$ and control $(n=28)$ groups. Statistically significant increases in sick-role behavior, basic psychological needs, and self-efficacy were found in the experimental group $(p<0.001)$. Physiological parameters were maintained within the normal ranges in the experimental group, and the number of non-adherent patients decreased, although the change was not statistically significant. Conclusions: The mobile-app-based self-management program developed in this study increased the sickrole behavior, basic psychological needs, and self-efficacy of elderly hemodialysis patients, while physiological parameters were maintained within the normal range. Future studies are needed to develop management systems for high-risk hemodialysis patients and family-sharing apps to manage non-adherent patients.

Keywords: Aged, Renal Dialysis, Mobile Applications, Personal Health Services, Self-Management

Submitted: January 17, 2020

Revised: 1st, March 29, 2020; 2nd, April 22, 2020

Accepted: April 22, 2020

\section{Corresponding Author}

Myonghwa Park

College of Nursing, Chungnam National University, 266, Munhwaro, Jung-gu, Daejeon 35015, Korea. Tel: +82-42-580-8328, E-mail: mhpark@cnu.ac.kr (https://orcid.org/0000-0002-0329-0010)

This is an Open Access article distributed under the terms of the Creative Commons Attribution Non-Commercial License (http://creativecommons.org/licenses/bync/4.0/) which permits unrestricted non-commercial use, distribution, and reproduction in any medium, provided the original work is properly cited.

(C) 2020 The Korean Society of Medical Informatics

\section{Introduction}

Most elderly people with chronic kidney disease choose to receive hemodialysis. The increasing number of elderly hemodialysis patients and the associated cost of dialysis make quality control necessary [1]. Death due to cardiovascular complications occurs more frequently in elderly hemodialysis patients than in younger patients [2], and this is associated with hyperphosphatemia, hyperkalemia, and excessive interdialytic weight gain rate (IWGR). Serum phosphorus is an indicator of dietary adherence, and serious complications can result if hyperphosphatemia persists. Despite improvements in renal replacement therapy, regular hemodialysis 
alone cannot effectively remove phosphate from the blood [3], so hemodialysis, phosphate binders, and restriction of phosphate-rich food must continue for controlling the serum phosphate level [4]. Hyperkalemia can cause severe arrhythmias, such as ventricular fibrillation and cardiac arrest. The frequency of emergency room visits increases significantly when the serum potassium level exceeds a certain threshold, such as $6.0 \mathrm{mEq} / \mathrm{L}$ [5]. Therefore, the potassium intake must be restricted to prevent hyperkalemia. Dietary education and prescription of potassium-binding drugs are reported to be more effective in reducing high levels of serum potassium than other methods [6], so interventions have been developed to promote patient adherence. The IWGR can be used as an adherence indicator for diet and nutrition [7]. An increase in the IWGR of more than $5.7 \%$ is associated with high mortality and is regarded as indicating non-adherence [8]. However, elderly hemodialysis patients are more prone to malnutrition than younger patients, leading to worse immunological function and increased mortality [9], so dietary restriction should be minimized in the elderly. Moreover, protein intake should not be reduced by excessive dietary restriction aimed at controlling hyperphosphatemia or hyperkalemia.

Sick-role behavior refers to the extent to which a patient's behavior matches the prescribed treatment plan, and clinical prescriptions can be divided into adherence and non-adherence [10]. The serum phosphate and potassium levels as well as the IWGR are the main parameters used to indicate physiological adherence of sick-role behavior in hemodialysis patients. Self-determination theory explains human motives and conceptualizes human behavior on a continuum ranging from purely externally synchronized behavior to fully internally synchronized behavior. The intrinsic motivation is the innate motivation that appears voluntarily from the basic psychological needs for autonomy, competence, and relationships [11]. The sick-role behavior of elderly hemodialysis patients must change from their previous lifestyle and behavior, and these patients must greatly restrict their actions while performing self-determination behaviors based on their own decisions [11]. Achieving this requires the induction of intrinsic motivation and internalization of externally synchronized actions to reach integrated coordination, and to develop interventions that can meet the three basic needs [12].

Self-efficacy refers to the expectations and beliefs that an individual has the ability to do something successfully. In hemodialysis patients, self-efficacy is an important factor influencing the motivation for health-promotion behavior [13].
Health providers should recognize that self-efficacy is a key factor in successfully starting and maintaining health-related behavioral changes and seeking a supportive approach [14].

While some apps have been developed and are being utilized as essential tools for maintaining health, most of the related studies have involved middle-aged populations [1517], and these apps are not ideal for application to elderly patients in clinical settings. However, it has been shown that the intention to accept smartphone-based health management approaches among elderly and chronic-disease patients is high, and an important reason for using the Internet is to obtain health-related information [18]. Moreover, since mobile devices can provide customized messages tailored to individual characteristics through personalized media, they are easier than a computer to use independently. Therefore, the purpose of this study was to develop a mobile-app-based self-management support program and to determine its effects on sick-role behavior adherence, basic psychological needs, self-efficacy, and physiological parameters of elderly hemodialysis patients.

\section{Methods}

\section{Design}

This study had a nonequivalent control-group non-synchronized design.

\section{Samples}

This study included patients receiving hemodialysis on an outpatient basis at the hemodialysis unit of Chungnam National University Hospital in Daejeon, South Korea who satisfied the following inclusion criteria and consented to participate in the study: aged 60 to 85 years, receiving continuous hemodialysis for more than 3 months, able to use a mobile-app without hindrance from a visual or hearing disorder, receiving hemodialysis more than eight times per month (twice weekly), and can understand the content of the study. The following exclusion criteria were applied: mental illness with hospitalization, transferred to another hospital, received kidney transplant, or did not provide voluntary consent.

The required sample size was calculated using the $G^{\star}$ Power 3.1 program based on a previous study [14] that applied interventions and tested the effects on hemodialysis patients at a significance level $(\alpha)$ of 0.05 , an effect size of 0.4 , a statistical power $(1-\beta)$ of 0.80 , and using repeated-measures ANOVA. The number of samples required was 40 in total (20 in each of the two measurement groups), and so data collec- 
tion was carried out with the goal of recruiting 30 people per group based on consideration of the possible dropout rate.

The primary investigator reviewed the Electronic Medical Records (EMRs) and conducted a baseline survey on the use of mobile communication devices. Seventy-four patients who satisfied the inclusion criteria were contacted, and 30 subjects who consented to participate in the study were first enrolled in the control group to prevent the spread of the information about the study. After 2 deaths, 28 subjects (6.7\% dropout rate) were included in the final data analysis as the control group. The experimental group initially comprised 30 subjects who consented to participate in the study, but after 2 subjects were transferred to another hospital, 28 subjects $(6.7 \%$ dropout rate) were included in the final data analysis as the experimental group (Figure 1).

\section{Measurements}

1) Sick-role behavior adherence

Sick-role behavior adherence was assessed using 16 questions on diet, medication, exercise, dialysis plan, and vascular access management in hemodialysis patients. The questions were scored on a 5-point Likert scale, with a higher score indicting greater adherence. Cronbach's $\alpha$ for the reliability of the tool was 0.81 in a previous study [19] and 0.74 in this study.

\section{2) Basic psychological needs}

Basic psychological needs were assessed using 18 questions scored on a 5-point Likert scale, with higher scores indicating greater needs. Cronbach's $\alpha$ for the reliability of the tool was 0.78 in a previous study [20] and 0.91 in this study.

\section{3) Self-efficacy}

A self-efficacy measurement tool was developed by Kim and Kim [21] for hemodialysis patients based on a 4-point Likert scale, where higher scores indicate higher self-efficacy. Cronbach's $\alpha$ for the reliability of the tool was 0.81 in a previous study [21] and 0.80 in this study.

\section{4) Serum phosphate}

A serum phosphate level of $>5.5 \mathrm{mg} / \mathrm{dL}$ measured before beginning hemodialysis was considered to indicate non-adherence based on the 2015 chronic kidney disease-mineral and bone disorder guideline [22].

\section{5) Serum potassium}

A serum potassium level of $>6.0 \mathrm{mEq} / \mathrm{L}$ measured before beginning hemodialysis was considered to indicate nonadherence [5].

6) Interdialytic weight gain rate

A mean ratio of the dry weight measured in an interim test day to the weight gain prior to hemodialysis (measured four times) of $>5.7 \%$ during the last 4 weeks was considered to indicate non-adherence [23].

\section{7) General characteristics}

Sex, age, comorbidity, living arrangement, duration of hemodialysis, job, education, monthly income, and health insurance type were also determined for each subject (Table 1).

\section{Procedures}

1) Development of the program

A preliminary survey was done on smartphone use by the

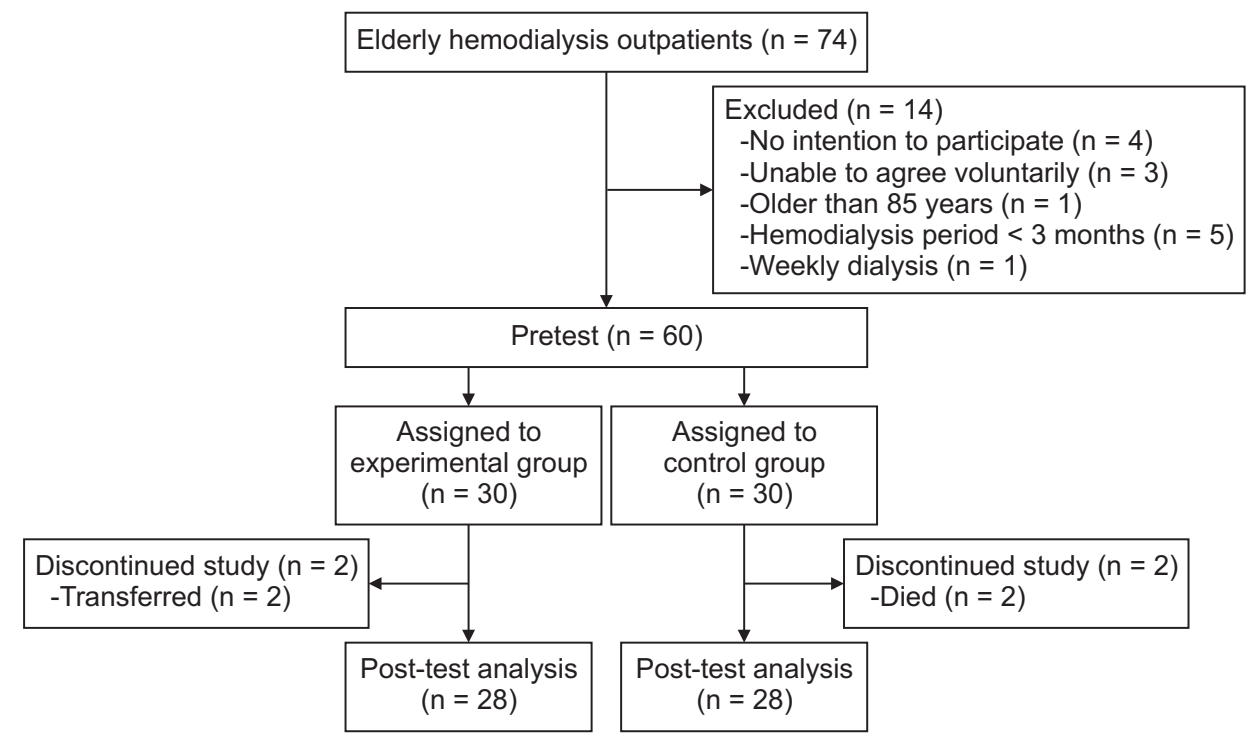

Figure 1. Flow chart of the study. 
Table 1. Homogeneity test of general characteristics $(n=56)$

\begin{tabular}{|c|c|c|c|c|}
\hline Variable & Experimental group $(n=28)$ & Control group $(n=28)$ & $\chi^{2}$ or $t$ & $p$-value \\
\hline Gender & & & 0.29 & 0.584 \\
\hline Male & $18(64.3)$ & $16(57.1)$ & & \\
\hline Female & $10(35.7)$ & $12(42.9)$ & & \\
\hline Age (yr) & & & 4.46 & 0.068 \\
\hline $60-75$ & $24(85.7)$ & $17(60.7)$ & & \\
\hline $75-85$ & $4(14.3)$ & $11(39.3)$ & & \\
\hline Education & & & 2.50 & 0.205 \\
\hline$\leq$ Elementary school & $4(14.3)$ & $9(32.1)$ & & \\
\hline$\geq$ Middle school & $24(85.7)$ & $19(67.9)$ & & \\
\hline \multicolumn{5}{|l|}{ Spouse } \\
\hline With spouse & $27(96.4)$ & $24(85.7)$ & 1.97 & 0.160 \\
\hline Without spouse & $1(3.6)$ & $4(14.3)$ & & \\
\hline Living arrangement & & & 2.42 & 0.297 \\
\hline Alone & $1(3.6)$ & $3(10.7)$ & & \\
\hline With spouse only & $23(82.1)$ & $18(64.3)$ & & \\
\hline With family & $4(14.3)$ & $7(25.0)$ & & \\
\hline Comorbidity $^{\mathrm{a}}$ & & & - & - \\
\hline Diabetes mellitus & $17(60.7)$ & $19(67.9)$ & & \\
\hline Hypertension & $24(85.7)$ & $21(75.0)$ & & \\
\hline Heart disease & $6(21.4)$ & $10(35.7)$ & & \\
\hline Neuralgia/arthritis & $2(7.1)$ & $7(25.0)$ & & \\
\hline Others & $6(21.4)$ & $14(50.0)$ & & \\
\hline Job & & & & $0.491^{b}$ \\
\hline Employed & $2(7.1)$ & - & & \\
\hline Unemployed & $26(92.9)$ & $28(100.0)$ & & \\
\hline Duration of hemodialysis (yr) & $4.23 \pm 3.32$ & $5.33 \pm 5.43$ & -0.90 & 0.367 \\
\hline$<1$ & $5(17.9)$ & $3(10.7)$ & 2.16 & 0.706 \\
\hline$\geq 1$ and $<5$ & $11(29.3)$ & $11(50.0)$ & & \\
\hline$\geq 5$ and $<10$ & $10(35.7)$ & $7(25.0)$ & & \\
\hline$\geq 10$ & $2(7.1)$ & $4(14.3)$ & & \\
\hline Monthly income (10,000 Korean won) & & & 0.73 & 0.693 \\
\hline$<100$ & $13(46.4)$ & $15(53.6)$ & & \\
\hline$\geq 100$ and $<200$ & $2(7.1)$ & $3(10.7)$ & & \\
\hline$\geq 200$ & $13(46.4)$ & $10(35.7)$ & & \\
\hline Insurance & & & 0.22 & 0.639 \\
\hline Medical aid & $2(7.1)$ & $3(10.7)$ & & \\
\hline Medical insurance & $26(92.9)$ & $25(89.3)$ & & \\
\hline
\end{tabular}

Values are presented as number $(\%)$ or mean \pm standard deviation.

${ }^{a}$ Multiple response.

${ }^{\mathrm{b}}$ Fisher exact test. 
included elderly patients. The findings showed that most of them were using a social network service, such as KakaoTalk, and they had a considerable amount of experience in receiving app information from their families. However, none of them had used the app from Chungnam National University Hospital.

Using the hospital information system for patients, we developed a feedback system for blood test results with visual graphics in the app. Dry weight was shown with the body mass index, and the change in weight gain was represented using arrows and numbers to enhance the visual presentation of information. A messaging service was utilized through message templates. Standardized messages were developed in a reference review related to health communications and in consultation with two nephrologists and two experienced nurses. The department of medical information and legal team were consulted regarding the processing of personal information. A delivery program using the blood test prescription code of the hemodialysis unit was developed to send regular single-screen notification messages containing information on interim blood tests and nonadherence (Figure 2).

A program validity test was performed by nine professionals. The content validity index was $>0.90$. The developed app was tested on five elderly patients to determine its usability prior to beginning the main intervention.

\section{2) Intervention}

To enhance app utilization, we provided the participants with 1 week of education on how to use their smartphones. Re-education was conducted 4 weeks later. Both the experimental and control groups underwent regular blood tests and health checkups by a physician, and were provided with education on diet and interim blood tests.

The usual care (physician checkups and education on diet and medication) was provided to the experimental and control groups. Appointment reminder messages for regular blood tests were sent to the experimental group. The interdialytic weight gain was entered by the patient every week, and feedback messages were provided that comprised test results and information on complications and dietary restrictions. In particular, more information on symptoms was provided when patients should visit the emergency room. The blood test results in the regular monitoring were sent via smartphone, and the changes in the test results were displayed in graphs to make it easy for patients to understand the relationship between the changes and adherence to medication and diet (Figures 2, 3). All interventions were provided by the researcher who had sufficient clinical experience of more than 8 years in a hemodialysis unit.

\section{Statistical Analysis}

The collected data were analyzed using SPSS version 22.0 for Windows (IBM, Armonk, NY, USA). The general characteristics of each subject were presented as number and percentage values and also mean \pm standard deviation values. The homogeneity in the experimental and control groups was analyzed using the $\chi^{2}$-test, independent $t$-test, and Fisher exact test. The conformity with normality of research variables was confirmed using the Shapiro-Wilk test, and Cronbach's $\alpha$ was used to assess the reliability of the measurement tools. Repeated-measures ANOVA was used to assess differences between measured variables, and covariance analysis was used for hypothesis testing of basic psychological needs,

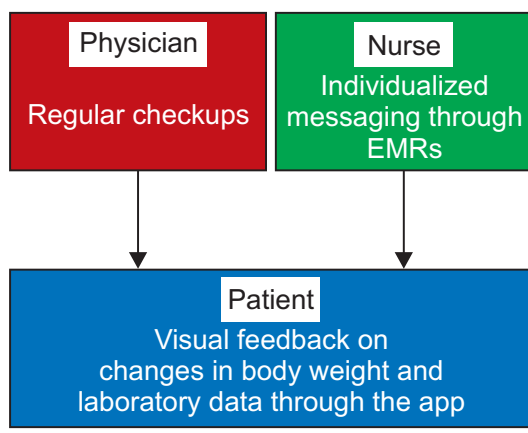

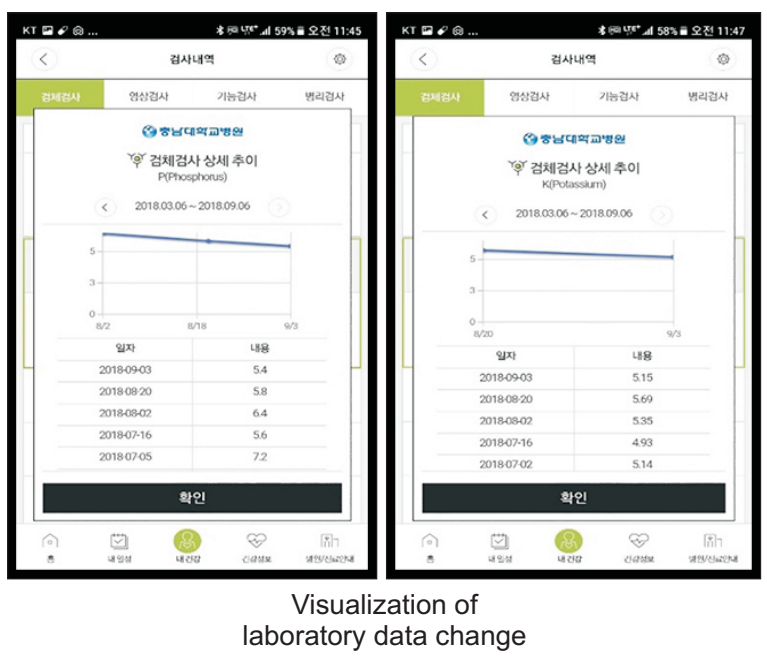

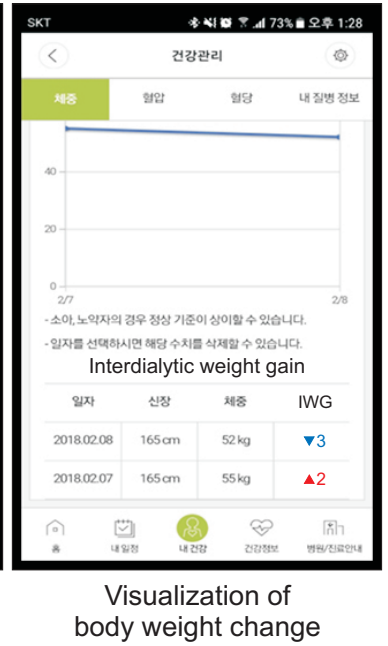

Figure 2. Intervention process. 


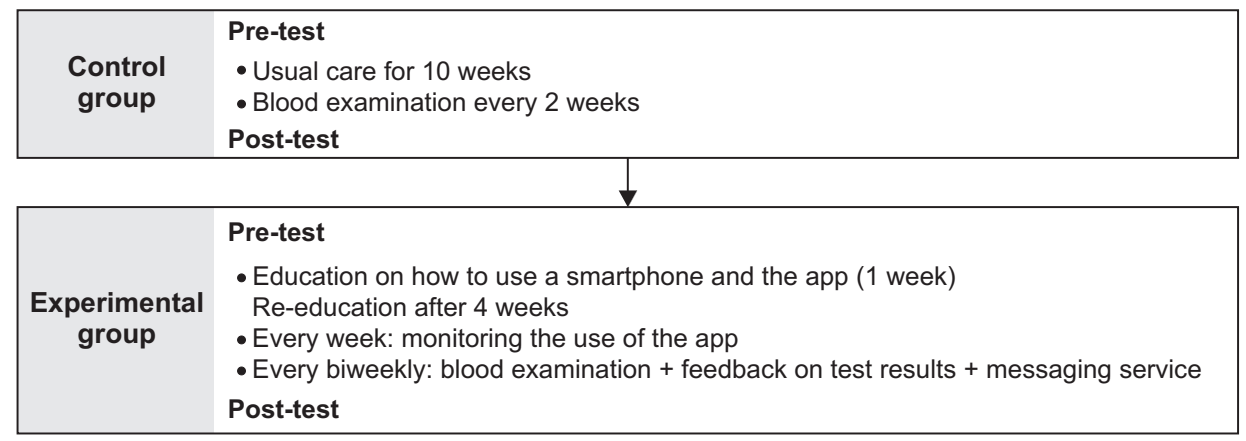

Figure 3. Study procedure.

Table 2. Homogeneity test of research variables $(n=56)$

\begin{tabular}{lcccc}
\hline \multicolumn{1}{c}{ Variable } & Experimental group $(\mathrm{n}=\mathbf{2 8})$ & Control group $(\mathrm{n}=\mathbf{2 8})$ & $t$ & $p$-value \\
\hline Sick role behavior & $56.53 \pm 8.63$ & $54.21 \pm 8.32$ & 1.02 & 0.311 \\
Basic psychological need & $65.42 \pm 7.35$ & $56.75 \pm 12.03$ & 3.25 & 0.002 \\
Self-efficacy & $24.67 \pm 4.62$ & $22.57 \pm 5.98$ & 1.47 & 0.146 \\
Phosphate (mg/dL) & $4.30 \pm 1.17$ & $4.01 \pm 1.16$ & 0.94 & 0.347 \\
Potassium (mEq/L) & $5.04 \pm 0.62$ & $4.82 \pm 0.80$ & 1.16 & 0.250 \\
Interdialytic weight gain (\%) & $3.91 \pm 1.72$ & $3.60 \pm 1.22$ & 0.78 & 0.435 \\
\hline
\end{tabular}

Values are presented as mean \pm standard deviation.

which showed differences in the pre-test homogeneity of the research variables because the pre-test basic psychological needs value was processed as a covariate. The homogeneity of dispersion was verified by Mauchly's sphericity test, and when the hypothesis of the sphericity test was not satisfied, the Greenhouse-Geisser procedure was used to correct the number of degrees of freedom. The McNemar test was used to compare changes in the 10-week pre-test and post-test adherence and non-adherence groups.

\section{Ethical Considerations}

Data collection was conducted at Chungnam National University Hospital, and was approved by the Institutional Review Board prior to commencement (No. CNUH 201802-018). Pre- and post-surveys were conducted at the hemodialysis unit by the investigator. If a subject asked or found it difficult to read, the interviewer read the questionnaire, and the subject recorded the response. A visualization measurement tool was used to increase the accuracy of understanding subjective parameters. Information related to disease characteristics of each subject was collected through medical records.

\section{Results}

\section{Homogeneity Testing of the General Characteristics and Dependent Variables}

The general characteristics did not differ significantly between the two groups, thereby confirming homogeneity (Table 2). Other than basic psychological needs, there were no significant differences in the dependent variables between the two groups, and the homogeneity of the two groups was confirmed.

\section{Effects of the Program}

1) Hypothesis 1: There are intergroup differences in sick-role behavior, basic psychological needs, and self-efficacy over time

The sick-role behavior in the experimental group increased after 10 weeks of intervention, whereas that in the control group decreased after 10 weeks, with a statistically significant difference between the two groups $(F=38.33, p<0.001)$. Additionally, there were significant differences according to measurement time $(F=75.01, p<0.001)$ and in the interaction between the groups and measurement time $(F=112.05$, $p<0.001)$. Basic psychological needs in the experimental group increased after 10 weeks of intervention, whereas those in the control group decreased after 10 weeks. When the pre-test score was processed as a covariate, it differed significantly between the two groups $(F=77.73, p<0.001)$. 
Self-efficacy in the experimental group increased after 10 weeks of intervention, whereas that in the control group decreased after 10 weeks, with a statistically significant difference between the two groups $(F=29.86, p<0.001)$. Additionally, there were significant differences according to measurement time $(F=29.42, p<0.001)$ and in the interaction between the groups and measurement time $(F=43.24$, $p<0.001$ ). Hypothesis 1 was therefore supported (Table 3 ).

2) Hypothesis 2: There are intergroup differences in physiological parameters over time

There were no significant differences in the serum phosphate level between the two groups ( $F=0.53, p=0.468$ ) according to measurement time $(F=0.12, p=0.987)$, or in the interaction between the groups and measurement time $(F=0.30, p=$ 0.909) (Table 4). There were also no significant differences in the serum potassium level between the two groups $(F=2.58$, $p=0.114)$ according to measurement time $(F=1.66, p=$ $0.144)$, or in the interaction between the groups and measurement time ( $F=0.74, p=0.589)$ (Table 4$)$. The IWGR did not differ significantly between the two groups $(F=0.08, p=$ 0.774). Also, there were no significant differences according to measurement time $(F=1.18, p=0.281)$ or in the interaction between the groups and measurement time $(F=2.21, p=$ 0.143 ). Hypothesis 2 was therefore rejected (Table 5).

Sub-hypothesis 2-1 was formulated as follows: there are differences between the experimental and control groups in physiological parameters over time according to adherence. Regarding the serum phosphate level in the experimental group, non-adherence decreased from 6 subjects at the pretest to three subjects at the post-test, while in the control group there were 3 non-adherent subjects at both the pretest and post-test. Regarding the serum potassium level in the experimental group, non-adherence decreased from 5 subjects at the pre-test to 2 subjects at the post-test, while in the control group the 1 non-adherent subject at the pretest increased to 3 at the post-test. Moreover, for the IWGR in the experimental group, non-adherence decreased from 5 subjects at the pre-test to 1 subject at the post-test, whereas there was no difference in the control group, with 1 non-adherent subject at both the pre-test and post-test. The McNemar test revealed no significant differences between the two groups regarding adherence for the serum phosphate level, serum potassium level, and the IWGR, so sub-hypothesis 2-1 was rejected (Table 6).

\section{Discussion}

The program developed in this study was found to exert significant effects on the basic psychological needs, self-efficacy, and sick-role behavior adherence of elderly hemodialysis patients, while no significant changes were found in serum phosphate and potassium levels. Additionally, although the IWGR decreased in the experimental group, this change was not statistically significant. Interventions applied to hemodialysis patients have been found to be helpful in improving their knowledge and attitude, but patients are often still not sufficiently motivated, and the increase in their knowledge does not always lead to sick-role behavior adherence [24]. This situation prompted the present study to develop a program to support self-management based on basic psychological needs, and this program was found to significantly affect the sick-role behavior adherence in the experimental group after the intervention. This may be attributed to the mobile-app-based self-management support program enabling healthcare providers to use self-determination to help

Table 3. Comparisons of research variables $(n=56)$

\begin{tabular}{|c|c|c|c|c|c|c|c|}
\hline \multirow{2}{*}{ Variable } & \multicolumn{2}{|c|}{ Experimental group $(n=28)$} & \multicolumn{2}{|c|}{ Control group $(n=28)$} & \multirow{2}{*}{ Sources } & \multirow{2}{*}{$F$} & \multirow{2}{*}{$p$-value } \\
\hline & Pre-test & Post-test & Pre-test & Post-test & & & \\
\hline \multirow[t]{3}{*}{ Sick role behavior } & $56.53 \pm 8.63$ & $72.96 \pm 5.56$ & $54.21 \pm 8.32$ & $52.57 \pm 7.36$ & Group & 38.33 & $<0.001$ \\
\hline & & & & & Time & 75.01 & $<0.001$ \\
\hline & & & & & Group $\times$ Time & 112.05 & $<0.001$ \\
\hline \multirow{3}{*}{$\begin{array}{l}\text { Basic psychological } \\
\text { need }\end{array}$} & $65.42 \pm 7.35$ & $76.96 \pm 7.21$ & $56.75 \pm 12.03$ & $54.42 \pm 10.59$ & - & $77.73^{\mathrm{a}}$ & $<0.001$ \\
\hline & & & & & - & - & - \\
\hline & & & & & - & - & - \\
\hline \multirow[t]{3}{*}{ Self-efficacy } & $24.67 \pm 4.62$ & $31.75 \pm 3.65$ & $22.57 \pm 5.98$ & $21.89 \pm 3.99$ & Group & 29.86 & $<0.001$ \\
\hline & & & & & Time & 29.42 & $<0.001$ \\
\hline & & & & & Group $\times$ Time & 43.24 & $<0.001$ \\
\hline
\end{tabular}

${ }^{a}$ ANCOVA with pre-test value as covariate. 


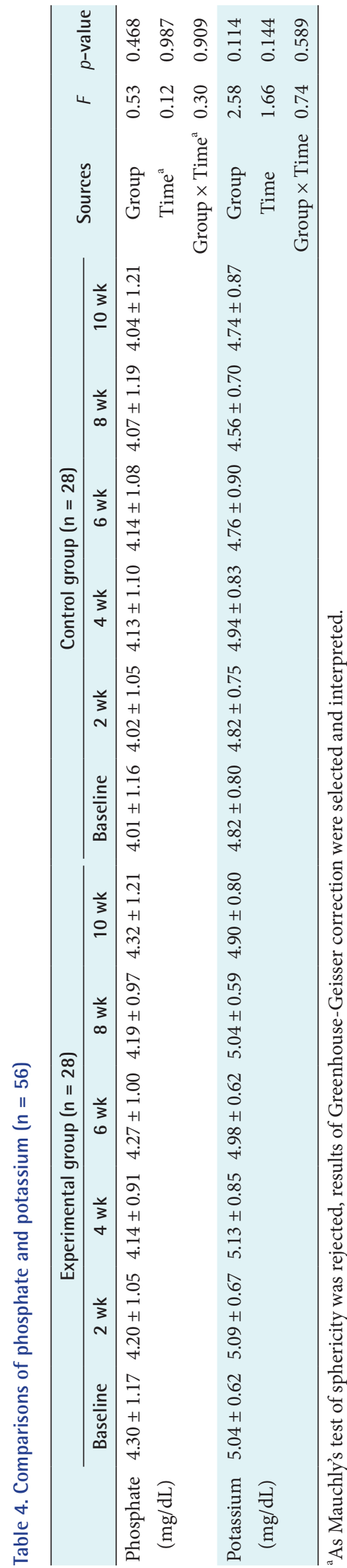

patients to take care of themselves [11]. Our results align with those of previous studies that showed that good health management, such as blood glucose control and weight loss, was associated with greater patient autonomy [25].

The serum phosphate level in the present experimental group did not change significantly from before the intervention ( $4.30 \pm 1.17$ points) to after the intervention ( $4.32 \pm 1.10$ points). This result may be attributed to interventions aimed at maintaining the protein intake of hemodialysis patients, supporting physicians' prescriptions, and encouraging the intake of foods with higher protein content than phosphate content (except where the serum phosphate level was already $>5.5 \mathrm{mg} / \mathrm{dL}$ ).

The serum potassium level in the experimental group also did not differ significantly between before and after the intervention (5.04 \pm 0.62 vs $4.90 \pm 0.80$ points). It is believed that diet should be restricted only in cases in which the potassium level is elevated to $>6.0 \mathrm{mEq} / \mathrm{L}$, but considering the various causes of potassium elevation, the physician's prescription was not changed and the patients managed their diet themselves. Continuous evaluation of and education provision to elderly hemodialysis patients is necessary considering the vegetable-based traditional eating habits of the elderly, their consumption of seasonal fruits, the various types of medicines they take, and their generally low adherence.

The IWGR in both groups remained within the normal range of $4 \%$, and the results for physiological parameters were also within the acceptable ranges, which contrasts with the findings of previous studies [26]. A study that compared the weight gains of hemodialysis patients according to the season found that the gain was lowest in July [27]. Thus, further studies should be conducted from March to early August to correctly evaluate the effects of the present program on the IWGR. Moreover, it is also possible that recognizing the IWGR individually and maintaining the food intake within the acceptable range affected the results. Moreover, the duration of hemodialysis was 4 to 5 years in all subjects, so the prolonged experience of receiving dietary education from physicians might also have affected the results.

The present mobile-app-based self-management program also improved self-efficacy. Similarly, self-efficacy was found to be significantly increased by a program utilizing a dietary management app [26] and a hemodialysis self-management app [28].

The numbers of non-adherent subjects-in terms of serum phosphate level, serum potassium level, and the IWGR-decreased in the experimental group. A previous study that as- 
Table 5. Comparisons of interdialytic weight gain $(n=56)$

\begin{tabular}{|c|c|c|c|c|c|c|c|}
\hline \multirow{2}{*}{ Variable } & \multicolumn{2}{|c|}{ Experimental group $(n=28)$} & \multicolumn{2}{|c|}{ Control group $(\mathrm{n}=28)$} & \multirow{2}{*}{ Sources } & \multirow{2}{*}{$F$} & \multirow{2}{*}{$p$-value } \\
\hline & Pre-test & Post-test & Pre-test & Post-test & & & \\
\hline \multirow{3}{*}{$\begin{array}{l}\text { Interdialytic weight } \\
\text { gain }(\%)\end{array}$} & $3.91 \pm 1.72$ & $3.54 \pm 1.21$ & $3.60 \pm 1.22$ & $3.65 \pm 1.34$ & Group & 0.08 & 0.774 \\
\hline & & & & & Time & 1.18 & 0.281 \\
\hline & & & & & Group $\times$ Time & 2.21 & 0.143 \\
\hline
\end{tabular}

${ }^{a}$ ANCOVA with pre-test value as covariate.

Table 6. Change in physiological parameters after the program

\begin{tabular}{|c|c|c|c|c|c|c|c|c|c|c|}
\hline & \multicolumn{5}{|c|}{ Experimental group $(\mathrm{n}=28)$} & \multicolumn{5}{|c|}{ Control group $(n=28)$} \\
\hline & \multicolumn{2}{|c|}{ Adherence } & \multicolumn{2}{|c|}{ Non-adherence $^{a}$} & \multirow{2}{*}{$p$-value } & \multicolumn{2}{|c|}{ Adherence } & \multicolumn{2}{|c|}{ Non-adherence $^{a}$} & \multirow{2}{*}{$p$-value } \\
\hline & Pre-test & Post-test & Pre-test & Post-test & & Pre-test & Post-test & Pre-test & Post-test & \\
\hline Phosphate (mg/dL) & $22(78.6)$ & $25(89.3)$ & $6(21.4)$ & $3(10.7)$ & 0.453 & $25(89.3)$ & $25(89.3)$ & $3(10.7)$ & $3(10.7)$ & 1.000 \\
\hline Potassium (mEq/L) & $23(82.1)$ & $26(92.9)$ & $5(17.9)$ & $2(7.1)$ & 0.453 & $27(96.4)$ & $25(89.3)$ & $1(3.6)$ & $3(10.7)$ & 0.625 \\
\hline $\begin{array}{l}\text { Interdialytic weight } \\
\text { gain }(\%)\end{array}$ & $23(82.1)$ & $27(96.4)$ & $5(17.9)$ & $1(3.6)$ & 0.219 & $27(96.4)$ & $27(96.4)$ & $1(3.6)$ & $1(3.6)$ & 1.000 \\
\hline
\end{tabular}

Values are presented as number (\%) by McNemar test.

${ }^{a}$ Criteria for non-compliance: phosphate $\geq 5.50 \mathrm{mg} / \mathrm{dL}$, potassium $\geq 6.0 \mathrm{mEq} / \mathrm{L}$, and interdialytic weight gain $\geq 5.7 \%$.

sessed the effects of an individual education program in nonsubjects found that non-adherence of the serum potassium level and the IWGR decreased while there was no decrease in the non-adherence of the serum phosphate level [29]. However, that study only included non-adherent patients. In contrast, the present study found that the proportion of nonadherent subjects was low, and that subjects self-monitoring their physiological parameters and interpreting their significance led to improvements, while the self-management behavior and parameters were within the normal ranges.

When implementing an intervention, variations in individual characteristics and proficiency of device use must be considered to ensure that the mobile app is utilized effectively [17]. Therefore, it is necessary to educate subjects about device use and prepare a support system when planning an intervention. Additionally, it is necessary to consider ways to actively utilize the social support system of the patient, such as the family, because families can provide a supportive learning environment for mobile usage, especially among the elderly. Regarding subject selection, previous studies involving clinical settings had limitations of including subjects with low mean ages [15-17], whereas the present study was meaningful in attempting to provide a mobile-app-based self-management support program to elderly hemodialysis patients aged $>60$ years. The results of this study will be particularly useful as the number of elderly hemodialysis patients increases concomitantly with the global aging popula- tion.

This study was subject to some limitations. Due to the high risk of contamination of the study subjects, blinding of the patients was not possible using the nonequivalent control group non-synchronized design. The long-term effects of this program are also unknown due to the short study duration of only 10 weeks.

Devices and programs need to be developed to enhance the involvement of healthcare providers before risk symptoms appear in patients [16]. Furthermore, the use of automated systems will enable effective personalized health management, which will help to reduce medical costs in the long term. Future studies are needed to develop high-risk hemodialysis patient management systems and family-sharing apps to manage non-adherent patients.

\section{Conflict of Interest}

No potential conflict of interest relevant to this article was reported.

\section{ORCID}

Youngsoon Min (http://orcid.org/0000-0002-4878-0099)

Myonghwa Park (http://orcid.org/0000-0002-0329-0010) 


\section{References}

1. Jin DC, Shin Y, Kim M, Kang M, Won E, Yang K. Analysis of hemodialysis therapy variation associated with periodic hemodialysis quality assessment by government in Korea. Korean J Med 2018;93(2):194-205.

2. Kalantar-Zadeh K, Regidor DL, Kovesdy CP, Van Wyck D, Bunnapradist S, Horwich TB, et al. Fluid retention is associated with cardiovascular mortality in patients undergoing long-term hemodialysis. Circulation 2009; 119(5):671-9.

3. Cupisti A, Gallieni M, Rizzo MA, Caria S, Meola M, Bolasco P. Phosphate control in dialysis. Int J Nephrol Renovasc Dis 2013;6:193-205.

4. Ketteler M, Block GA, Evenepoel P, Fukagawa M, Herzog CA, McCann L, et al. Executive summary of the 2017 KDIGO Chronic Kidney Disease-Mineral and Bone Disorder (CKD-MBD) Guideline Update: what's changed and why it matters. Kidney Int 2017;92(1):26-36.

5. Brunelli SM, Du Mond C, Oestreicher N, Rakov V, Spiegel DM. Serum potassium and short-term clinical outcomes among hemodialysis patients: impact of the long interdialytic interval. Am J Kidney Dis 2017;70(1):21-9.

6. Karaboyas A, Zee J, Brunelli SM, Usvyat LA, Weiner DE, Maddux FW, et al. Dialysate potassium, serum potassium, mortality, and arrhythmia events in hemodialysis: results from the Dialysis Outcomes and Practice Patterns Study (DOPPS). Am J Kidney Dis 2017;69(2):26677.

7. Lopez-Gomez JM, Villaverde M, Jofre R, RodriguezBenitez P, Perez-Garcia R. Interdialytic weight gain as a marker of blood pressure, nutrition, and survival in hemodialysis patients. Kidney Int Suppl 2005;(93):S638.

8. Saran R, Bragg-Gresham JL, Rayner HC, Goodkin DA, Keen ML, Van Dijk PC, et al. Nonadherence in hemodialysis: associations with mortality, hospitalization, and practice patterns in the DOPPS. Kidney Int 2003;64(1): 254-62.

9. Akner G, Cederholm T. Treatment of protein-energy malnutrition in chronic nonmalignant disorders. Am J Clin Nutr 2001;74(1):6-24.

10. Becker $\mathrm{MH}$. The health belief model and sick role behavior. Health Educ Monogr 1974;2(4):409-19.

11. Ryan RM, Patrick H, Deci EL, Williams GC. Facilitating health behaviour change and its maintenance: interventions based on self-determination theory. Eur Health Psychol 2008;10(1):2-5.
12. Cho YM, Sung KW. The association of compliance with sick role behavior with satisfaction of basic psychological needs among hemodialysis patients. Korean J Health Promot 2012;12(1):58-65.

13. Song MR. The development and test of self-efficacy promotion program on self-care of hemodialysis patients. J Korean Acad Nurs 2000;30(4):1066-77.

14. Yun KS, Choi JY. Effects of dietary program based on self-efficacy theory on dietary adherence, physical indices and quality of life for hemodialysis patients. J Korean Acad Nurs 2016;46(4):598-609.

15. Griva K, Nandakumar M, Ng JH, Lam KF, McBain H, Newman SP. Hemodialysis Self-management Intervention Randomized Trial (HED-SMART): a practical lowintensity intervention to improve adherence and clinical markers in patients receiving hemodialysis. Am J Kidney Dis 2018;71(3):371-81.

16. Hayashi A, Yamaguchi S, Waki K, Fujiu K, Hanafusa N, Nishi T, et al. Testing the feasibility and usability of a novel smartphone-based self-management support system for dialysis patients: a pilot study. JMIR Res Protoc 2017;6(4):e63.

17. Lee J, Lee $H$. The effects of smart program for patients who underwent percutaneous coronary intervention (SP-PCI) on disease-related knowledge, health behavior, and quality of life: a non-randomized controlled trial. J Korean Acad Nurs 2017;47(6):756-69.

18. Kim MY, Kang YH, Jung DY, Lee GJ. Older adults' smart phone use and access to health information. J Qual Res 2013;14(1):13-22.

19. Choi YS. A study on the relationship between perceived social support, and compliance with Sick role behavior in elderly hemodialysis patients [dissertation]. Seoul, Korea: Hanyang University; 2008.

20. Jung YS. The relationships among basic psychological needs, sensation seeking, flow, and exercise addiction of leisure sports participants [dissertation]. Seoul, Korea: Sungkyunkwan University; 2010.

21. Kim JH, Kim MJ. The factors affecting quality of life of hemodialysis patients. Korean J Adult Nurs 1995;7(2): 299-312.

22. Hwang E, Choi BS, Oh KH, Kwon YJ, Kim GH. Management of chronic kidney disease-mineral and bone disorder: Korean working group recommendations. Kidney Res Clin Pract 2015;34(1):4-12.

23. Wong MM, McCullough KP, Bieber BA, Bommer J, Hecking M, Levin NW, e al. Interdialytic weight gain: trends, predictors, and associated outcomes in the Inter- 
national Dialysis Outcomes and Practice Patterns Study (DOPPS). Am J Kidney Dis 2017;69(3):367-79.

24. Park JS, Lee HS, Lee SY, Im HB, Oh HS. Relationship between knowledge and adherence to self-management guidelines, and influencing factors of adherence for hemodialysis patients. Korean J Rehabil Nurs 2011;14(1): 39-46.

25. Silva MN, Markland D, Minderico CS, Vieira PN, Castro MM, Coutinho SR, et al. A randomized controlled trial to evaluate self-determination theory for exercise adherence and weight control: rationale and intervention description. BMC Public Health 2008;8:234.

26. Song YB. The effect of application-based dietary selfmanagement program on biochemical indicators, selfefficacy and quality of life in hemodialysis patients [dis- sertation]. Seoul, Korea: Kyung Hee University; 2018.

27. Chung S, Kahng KW, Hong TW, Lee CH, Kang CM, Park CH. Seasonal variation of interdialytic weight gain in Korean hemodialysis patients. Korean J Nephrol 2003; 22(4):426-32.

28. Kyung JB. The effects on interdialytic weight gain, selfefficacy and self-care behavior in hemodialysis patient by using mobile application for self-management [dissertation]. Seoul, Korea: Hanyang University; 2016.

29. Lee SJ, Park HS, Han JH, Kim HJ, Seo MW, Choi EA, et al. Effects of an individualized educational program on knowledge, compliance and physiologic parameters in non-compliant hemodialysis patients. J Korean Clin Nurs Res 2009;15(3):5-16. 